\title{
Degradação parcial de 17ß-estradiol por cloração aplicada ao tratamento da água
}

\author{
Partial degradation of $17 \beta$-estradiol by the chlorination \\ applied to the treatment of water
}

\begin{abstract}
Renata de Oliveira Pereira
Doutora em Hidráulica e Saneamento. Professora do Departamento de Engenharia Sanitária e Ambiental da Universidade Federal de Juiz de Fora - Juiz de Fora (MG), Brasil.
\end{abstract}

Vivian Maria Carminato

Engenheira Ambiental. Mestranda vinculada ao Departamento de Hidráulica e Saneamento da Escola de Engenharia de São Carlos da Universidade de São Paulo - São Carlos (SP), Brasil.

\section{Eny Maria Vieira}

Doutora em Química Analítica. Professora do Instituto de Química de São Carlos da Universidade de São Paulo - São Carlos (SP), Brasil.

\section{Luiz Antonio Daniel}

Doutor em Engenharia Civil. Professor do Departamento de Hidráulica e Saneamento da Escola de Engenharia de São Carlos da Universidade de São Paulo São Carlos (SP), Brasil.

\section{Resumo}

O objetivo do estudo foi avaliar a degradação do hormônio 17ß-estradiol com doses de cloro e tempos de contato comumente utilizados em estações de tratamento de água. A aplicação de cloro levou a pequenas alterações na maioria das variáveis analisadas. A concentração inicial de $17 \beta$-estradiol influenciou a remoção pelo cloro, e observou-se que o aumento da dose de cloro aumentou a remoção do $17 \beta$-estradiol até a dose de 2 mg.L-1. A maior remoção de hormônio obtida foi de 99\%, e a inativação dos microrganismos indicadores, E. coli e coliformes totais foi de até 5,6 log para valores de CT maiores que $14 \mathrm{mg} \cdot \min \cdot \mathrm{L}^{-1}$

Palavras-chave: cloração; desinfecção; estrógeno; desregulador endócrino; hormônio; oxidação.

\section{Abstract}

The purpose of this study was to evaluate the degradation of the estrogen $17 \beta$-estradiol at doses of chlorine and contact times commonly used in water treatment plants. The application of chlorine led to minor changes in most parameters examined. The initial concentration of the hormone affected the removal by chlorine and was observed that increasing the dose of chlorine increased the removal of $17 \beta$-estradiol until the dose of 2 mg. $L^{-1}$. The highest removal of hormone obtained was $99 \%$, and inactivation of the indicator microorganisms, E. coli and total coliform was up to 5.6 log for values of CT greater than $14 \mathrm{mg} \cdot \mathrm{min} \cdot \mathrm{L}^{-1}$.

Keywords: chlorination; disinfection; estrogen; endocrine disrupting; hormone; oxidation.

\section{Introdução}

A presença de contaminantes recalcitrantes na hidrosfera, devido às atividades industrial e agrícola, tem levado a uma preocupação particular com a qualidade das águas superficiais, subterrâneas e marinhas. Entre os compostos que causam risco à saúde destacam-se os desreguladores endócrinos (DE), que são compostos químicos exógenos que interferem na atividade hormonal pela ação de receptores (MAIA e DEZOTTI, 2007). Tais substâncias podem ser de origem antrópica, também denominadas xenobióticos, ou de origem natural, tais como os fitoestrogênios.

Vários efeitos têm sido atribuídos à exposição a esses compostos, tais como a diminuição na quantidade de esperma, o aumento de 
câncer de mama em mulheres e o aumento de certas anormalidades no sistema reprodutivo humano (LAGANÀ et al., 2004). Em peixes, foram observados efeitos como feminização e reversão sexual, inibição do crescimento testicular, inibição da espermatogênese, decrescimento da capacidade de fertilização dos ovos pela redução da produção dos hormônios sexuais masculino e alteração no comportamento reprodutivo (MILL \& CHICHESTER, 2005; SUMPTER, 2005).

O estrogênio ovariano biologicamente mais potente é o $17 \beta$-estradiol (E2), que está relacionado ao desenvolvimento das características secundárias sexuais femininas e à reprodução. É um estrogênio natural, contudo manufaturado em grandes quantidades para ser usado em contraceptivos orais e para a reposição hormonal (IKEHATA et al., 2006). Por ser um potente estrogênio, o E2 pode causar alterações nos sistemas endócrinos de organismos vivos mesmo em baixas concentrações na água, da ordem de ng. L $^{-1}$ (SUMPTER, 2005).

Os estrogênios naturais são biotransformados, primeiramente no sistema hepático e parcialmente nos músculos, rins e gônadas, sendo degradados a compostos estrogênicos menos ativos conjugados a ácidos sulfúrico e glicurônico (GILMAN et al., 2003). Entretanto, a ocorrência de estrogênios "livres" em Estações de Tratamento de Esgoto (ETE) indica que os metabólitos de estrogênios são convertidos de volta a sua forma ativa durante o tratamento do esgoto. Alguns pesquisadores sugerem que a desconjugação ocorre durante o processo do tratamento do esgoto (CHEN e HU, 2009; TERNES et al., 1999). Logo, os hormônios naturais e sintéticos permanecem na sua forma ativa e podem contaminar os corpos receptores. Os estrogênios conjugados já foram encontrados no meio ambiente, como o estrona-3-sulfato detectado no rio Llobregat na Espanha, em concentração de 0,33 ng. L $^{-1}$ (RODRIGUEZ-MOZAZ et al., 2004). No Brasil, também foram detectados estradiol-17-glucoronideo em 11 amostras com média de 3,5 ng. $\mathrm{L}^{-1}$ e estradiol-3-sulfato em 2 amostras com média de 0,72 ng.L-1 (KUSTER et al., 2009).

A ineficiência na remoção de DE durante o tratamento de esgoto resulta na presença desses no efluente final (BIRKETT $\&$ LESTER, 2002). A degradação do E2 por sistemas aeróbios é de até $88 \%$ após 1 dia de detenção hidráulica e para sistemas anaeróbios é de até 50\% após 5 dias de detenção hidráulica (LEE \& LIU, 2002). Após serem lançados nos corpos receptores, os DE podem ser removidos por fotodegradação, contudo sua remoção é lenta, com meia-vida de 10 dias (JURGENS et al., 2002). Por conseguinte, a água pode estar contaminada e, se captada, torna-se uma fonte adicional de DE para os consumidores da água tratada (LEINSTER et al., 1981).

A presença de E2 em rios e na água para abastecimento humano é cada vez mais frequente. O E2 foi detectado em uma fonte de água nos EUA na concentração de 17 ng. $\mathrm{L}^{-1}$, em Taiwan na ordem de 1,4 a 33,9 ng. $\mathrm{L}^{-1}$ em águas superficiais, no Brasil no afluente de uma estação de tratamento de água (ETA) convencional na ordem de 3,0 a 9,1 ng. $\mathrm{L}^{-1}$ e na água de abastecimento humano com concentração de 0,78 a 1,48 ng. $L^{-1}$ e em mananciais de Belo Horizonte e São Paulo com concentrações de 1,5 a 36,8 ng. $\mathrm{L}^{-1}$ e 0,72 a 17,1 ng. $\mathrm{L}^{-1}$ respectivamente (BENOTTI et al., 2009; CHEN et al., 2007; GEROLIN, 2008; MIERZWA et al., 2009).

Aparentemente, os tratamentos envolvidos na etapa da clarificação não são efetivos na remoção ou transformação de compostos estrogênicos (SVENSON; ALLARD; MATS 2003). No estudo desenvolvido por Westerhoff et al. (2005), a remoção de E2 foi de 2\% após coagulação com sulfato de alumínio. Chen et al. (2007), após coagulação/floculação com sulfato de alumínio ( $5 \mathrm{mg} . \mathrm{L}^{-1}$ ), obtiveram remoção de 30 a 50\%. No mesmo estudo, após a filtração rápida, a remoção foi de 31 a 42\% (filtro apenas com areia). Para o 17ß-estradiol, o tratamento mais recomendado seria a remoção por carvão ativado, que tem uma elevada eficiência de remoção - 97\% após 4 h de tempo de contato com $5 \mathrm{mg} \cdot \mathrm{L}^{-1}$ de carvão ativado (WESTERHOFF et al., 2005). Contudo, é um tratamento caro e que necessita de um tratamento posterior para o resíduo gerado e, adicionalmente, o tempo de detenção de 4 h é elevado para ser utilizado em uma ETA.

A oxidação, por conseguinte, é um método utilizado nas ETA e pode ser usado para a remoção dos compostos estrogênicos. Pereira et al. (2011) compararam o uso de diferentes oxidantes para a transformação de compostos estrogênicos, indicando que os tratamentos que apresentam as maiores eficiências de remoção são o ozônio, íon ferrato e dióxido de cloro. Contudo, o cloro é amplamente utilizado como desinfetante e, por sua característica oxidante, pode remover compostos orgânicos ou converter compostos tóxicos em não tóxicos.

No trabalho de Westerhoff et al. (2005), alcançou-se uma eficiência de remoção próxima a 100\% com tempo de contato de 24 h, dose de cloro de 3,5 a 3,8 mg. $\mathrm{L}^{-1}$ e concentração inicial de E2 de 10-250 ng. $\mathrm{L}^{-1}$. No estudo de Alum et al. (2004), chegou-se a uma remoção de $99 \%$ de $\mathrm{E} 2$ com $1 \mathrm{mg} \cdot \mathrm{L}^{-1}$ de cloro e tempo de contato de 15 minutos, com uma concentração inicial elevada e limite de detecção do equipamento de 313 ng..-1. Nakamura et al. (2007), após a oxidação com cloro de $1 \mathrm{mg} \cdot \mathrm{L}^{-1}$ e tempo de contato de 15 minutos, chegaram a uma remoção de $70 \%$ para o estrona (E1). Portanto, doses de cloro entre 1 e 4 mg. L $^{-1}$ têm se mostrado eficiente para a remoção de compostos estrogênicos. Contudo, a cloração de compostos estrogênicos se mostra dependente do tempo de contato, alcançando eficiências elevadas de remoção depois de elevados tempos de contato (ALUM et al., 2004; NAKAMURA et al., 2007; PEREIRA et al., 2012; WESTERHOFF et al., 2005).

Portanto, o objetivo deste estudo foi avaliar a eficiência de remoção de $17 \beta$-estradiol da água de abastecimento por meio da oxidação com cloro empregando as doses e tempos de contato normalmente utilizados nas ETA no Brasil, considerando diferentes concentrações iniciais do estrógeno $17 \beta$-estradiol. 


\section{Metodologia}

\section{Padrões, soluções, equipamentos e condições analíticas}

Todos os solventes utilizados foram grau HPLC e o padrão $17 \beta$-estradiol - Sigma Reference Standard, fornecido pela Sigma Aldrich. Este foi adicionado a partir de solução estoque preparada diluindo-se o padrão de $17 \beta$-estradiol em metanol na concentração de $100 \mathrm{mg} \cdot \mathrm{L}^{-1}$. As concentrações de $17 \beta$-estradiol utilizadas neste estudo foram 100 e $1 \mu \mathrm{g} . \mathrm{L}^{-1}$ e $100 \mathrm{ng} . \mathrm{L}^{-1}$.

A identificação do E2 foi realizada utilizando-se cromatografia líquida de alta eficiência com detector de fluorescência (CLAEFLU) (Agilent Technologies - série 1200). A coluna utilizada foi a C18-PAH (Hidrocarbonetos Policíclicos Aromáticos) (250x4,6 mm, $5 \mu \mathrm{m}$ - Lichrospher), excitação a $230 \mathrm{~nm}$ e emissão a $306 \mathrm{~nm}$, volume de injeção de $20 \mu \mathrm{l}$, fluxo de $1 \mathrm{~mL} \cdot \mathrm{min}^{-1}$, temperatura $25^{\circ} \mathrm{C}$ e tempo de corrida de $10 \mathrm{~min}$. A fase móvel para concentrações acima de $1 \mu \mathrm{g} . \mathrm{L}^{-1}$ de E2 foi acetonitrila:água na proporção de 60:40 e para concentrações abaixo dessa foi de 48:52 (acetonitrila:água).

Para concentração de E2 abaixo de $1 \mu$ g. L $^{-1}$, foi necessário concentrar as amostras para posterior detecção. $O$ procedimento para preparação da amostra consistiu na extração em fase sólida com cartucho SPE-C18 (Accubond - $500 \mathrm{mg}$ ). O volume de amostra utilizado para extração foi $200 \mathrm{~mL}$, sendo o procedimento de extração adaptado de Ternes et al. (1999), no qual incluiu-se a etapa de clean-up com $10 \mathrm{~mL}$ da solução metanol:água deionizada na proporção de 1:9, eluição dos analitos com $4 \mathrm{~mL}$ de acetona. Após a extração, o volume eluído foi seco em um fluxo de nitrogênio e o analito ressuspendido em $500 \mu \mathrm{L}$ de acetonitrila. A validação da metodologia foi realizada de acordo com normas estabelecidas pela RE nº 899, de 29 de maio de 2003, da Agência Nacional de Vigilância Sanitária (ANVISA, 2003). Os limites de detecção e quantificação do método foram de 40 e 50 ng. L $^{-1}$, respectivamente. As concentrações relatadas em concentrações menores que o LD e LQ foram obtidas por meio do procedimento de adição padrão, sendo portanto estimativas. Adicionou-se quantidade suficiente do padrão de E2 à amostra original até se obter concentração de 50 ng. $\mathrm{L}^{-1}$, que após passar por todo o procedimento analítico era feita a quantificação do E2. Para obter a concentração real da amostra, descontou-se da concentração detectada a quantidade de E2 que foi adicionada inicialmente, tornando-se a amostra passível de ser quantificada.

\section{Matriz utilizada e caracterização da água}

Para a realização dos ensaios, foi utilizada água de poço artesiano coletada antes da cloração. Toda a água utilizada foi previamente filtrada em membrana de mistura de ésteres $(0,45 \mu \mathrm{m})$ com intuito de eliminar os sólidos em suspensão na amostra. A escolha dessa matriz baseou-se no fato de se utilizar água com características semelhantes às de água para o abastecimento público, com turbidez e cor baixa. Após a coleta, a água era armazenada a $4^{\circ} \mathrm{C}$ (CETESB, 1987).

A cepa Escherichia coli ATCC 11229 foi utilizada para avaliar a desinfecção com cloro. A cultura de E. coli foi preparada a partir de repicagens da cepa original em meio TSA - Tripticase Soy Agar. A cultura, na fase de crescimento log, foi adicionada conjuntamente com o E2 no início de cada tratamento, sendo avaliada a sua inativação após a aplicação do cloro.

A água de estudo foi caracterizada antes e após a adição do cloro, compreendendo as variáveis alcalinidade, coliformes totais, condutividade, cor, E. coli (filtração em membrana, meio de cultura Chromocult Coliform Agar, Merk), pH e turbidez, utilizando os procedimentos descritos em APHA (2005). O E2 foi analisado por CLAE-FLU, conforme procedimento descrito anteriormente.

\section{Cloração}

Os ensaios com cloro foram realizados em batelada em béqueres de 2 litros sob agitação com gradiente de $60 \mathrm{~s}^{-1}$. O hipoclorito de sódio ( $\mathrm{NaOCl}$ ) foi utilizado como desinfetante e preparado a partir de uma solução padrão com concentração de 4 a $6 \%$. As doses aplicadas, em mg.L.'- foram de 0,5, 1,0, 2,0 e 5,0 com diferentes tempos de contato. Todos os ensaios foram realizados em triplicata. Para a quantificação do cloro livre e do cloro total, foi utilizado o método DPD colorimétrico 4500-Cl G (APHA, 2005). No fim do tratamento ( $2 \mathrm{~L}$ de amostra), foi adicionado 0,5 $\mathrm{mL}$ de metabissulfito de sódio 3\% $\left(\mathrm{Na}_{2} \mathrm{~S}_{2} \mathrm{O}_{3}\right)$ para eliminar o residual de cloro.

\section{Análise estatística}

O tratamento estatístico utilizado para avaliar diferenças significativas na degradação de E2 entre as doses de cloro e os tempos de contato foi o delineamento inteiramente casualizado com experimentos fatoriais (BERTHOUEX \& BROWN, 2002). Os ensaios utilizados para a análise estatística foram: dose de cloro de 0,5, 1 e $2 \mathrm{mg} \cdot \mathrm{L}^{-1} \mathrm{e}$ tempos de contato de 10 e $30 \mathrm{~min}$, com concentração inicial ( $\left.C_{\mathrm{o}}\right)$ de E2 de 100 e $1 \mu \mathrm{g} . \mathrm{L}^{-1}$, analisados separadamente.

\section{Resultados e Discussão}

\section{Caracterização da água antes e após os ensaios}

Houve pequenas variações em algumas das variáveis analisadas após a cloração. A cor e turbidez não foram alteradas, houve consumo de alcalinidade e aumento da condutividade devido à aplicação do hipoclorito de sódio e/ou formação de produtos (Tabela 1). Em geral, a aplicação de hipoclorito contribui para o aumento do $\mathrm{pH}$, o que de fato ocorreu no primeiro minuto. No entanto, de acordo com que ocorria a degradação do E2 com o aumento do tempo de contato, o pH diminuía, podendo ser devido à geração de produtos com caráter ácido ou à degradação de compostos e liberação de $\mathrm{H}^{+}$(Figura 1). Considerando somente os 
resultados das variáveis analisadas, observa-se que a água apresenta características de água tratada adequada ao consumo humano.

Para todos os CT (Concentração de cloro x Tempo de contato) analisados, a inativação de E. coli esteve acima de 3 log chegando ao máximo de 5,6 log. Apenas em 3 tratamentos (CT de 8, 10 e 14 mg.min..-1)

Tabela 1 - Variáveis analisadas antes e após os ensaios com cloro $\left(n_{1}=24\right)$.

\begin{tabular}{lcccc|} 
& \multicolumn{3}{c}{ Antes da cloração } & \multicolumn{3}{c|}{ Após a cloração } \\
\cline { 2 - 5 } Variável & média & DP & média & DP \\
\hline $\begin{array}{l}\text { Alcalinidade total } \\
\left(\text { mg.CaCO }_{3} \cdot \mathrm{L}^{-1}\right)\end{array}$ & 29,1 & 3,5 & 26,0 & 3,8 \\
\hline Condutividade $\left(\mu \mathrm{S} \cdot \mathrm{cm}^{-1}\right)$ & 59,6 & 8,9 & 145,6 & 30,8 \\
\hline Cor $(\mathrm{uC})$ & 0,6 & 1,6 & 0,5 & 1,3 \\
\hline $\mathrm{pH}$ & 7,1 & 0,2 & 6,7 & 0,1 \\
\hline Temperatura ${ }^{\circ} \mathrm{C}$ & 16,0 & 2,8 & 17,0 & 2,8 \\
\hline Turbidez $(\mathrm{UT})$ & 0,4 & 0,2 & 0,4 & 0,2 \\
\hline
\end{tabular}

DP: Desvio Padrão; $\mathrm{n}_{1}$ : número de amostras; as características da água na Tabela 1 são as médias obtidas de 24 análises referentes aos ensaios de cloração com doses de 0,5, 1,0 e 2,0 mg.L-1-1 e tempos de contato de 1, 4, 7, 10, 20 e 30 minutos.

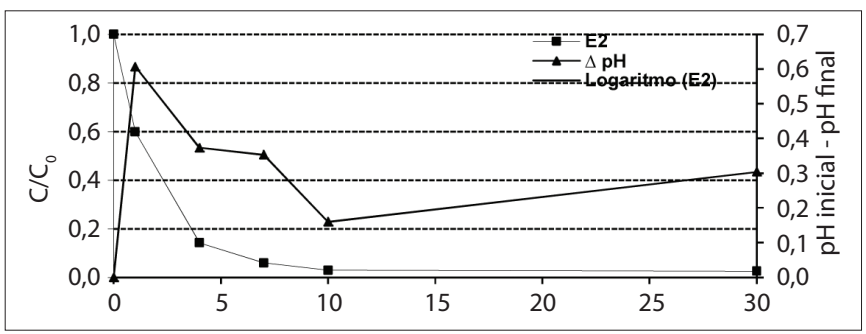

Figura 1 - Relação entre a concentração final e inicial de E2 e variação entre $0 \mathrm{pH}$ inicial e final em cada tempo de contato, $\mathrm{C}_{0}=100 \mu \mathrm{g} \cdot \mathrm{L}^{-1} \mathrm{de}$ E2 e $2 \mathrm{mgCl}_{2} \mathrm{~L}^{-1}$. a presença de E. coli foi confirmada. A presença de coliformes totais na água desinfetada foi mais frequente nos diferentes tratamentos. Até o CT de $10 \mathrm{mg}$.min. $\mathrm{L}^{-1}$, a presença de coliformes totais ocorreu nas três repetições, indicando a ineficiência de inativação desse microrganismo indicador pelo cloro. A partir de $10 \mathrm{mg} \cdot \mathrm{min} . \mathrm{L}^{-1}$, os coliformes totais estiveram presentes na água desinfetada de somente um dos tratamentos e não nas três repetições. Portanto, pode ser concluído que para CT menor do que 14 mg.min. L $^{-1}$ a desinfecção com cloro, avaliada pela inativação de coliformes totais, não foi eficiente nas condições em que os ensaios foram feitos.

\section{Oxidação de 17ß-estradiol com cloro}

\section{Análise estatística}

Nas duas concentrações iniciais de E2 de $100 \mu \mathrm{g} \mathrm{L}^{-1}$ e $1 \mu \mathrm{g} \mathrm{L}^{-1}$, avaliou-se que existe interação entre a dose de cloro e o tempo de contato ao nível de 5\% de significância, sendo portanto dependentes. Avaliou-se assim o comportamento de um fator em cada nível do outro (Tabela 2).

Para a concentração inicial de E2 de $100 \mu \mathrm{g} \cdot \mathrm{L}^{-1}$, concluiu-se que o tempo de contato não influenciou a remoção de E2, considerando a dose de cloro de $2 \mathrm{mg} . \mathrm{L}^{-1}$ e tempos de contato entre 10 e $30 \mathrm{mi}$ nutos. Entretanto, para as doses de cloro de 0,5 e $1 \mathrm{mg} . \mathrm{L}^{-1}$, o tempo de contato influenciou na degradação do E2. Por conseguinte, nessas condições, ou seja, $\mathrm{C}_{0}=100 \mu \mathrm{g} \cdot \mathrm{L}^{-1}$, o tratamento mais indicado seria a dose de cloro de $2 \mathrm{mg} . \mathrm{L}^{-1}$ com o tempo de contato de $10 \mathrm{~min}$ ou a dose de $0,5 \mathrm{mg} \cdot \mathrm{L}^{-1}$ durante $30 \mathrm{~min}$, para os quais a concentração residual de E2 foi 3,3 e 3,2 $\mu \mathrm{g} . \mathrm{L}^{-1}$, respectivamente (Figura 2A).

Tabela 2 - Remoção de 17 $\beta$-estradiol em diferentes condições de cloração $\left(\mathrm{n}_{2}=3\right)$.

\begin{tabular}{|c|c|c|c|c|c|}
\hline $\begin{array}{l}\text { Concentração inicial de } \\
\text { E2 } \\
\left(\mu \mathrm{g} \cdot \mathrm{L}^{-1}\right)\end{array}$ & $\begin{array}{l}\text { Tempo de contato } \\
\text { (min) }\end{array}$ & $\begin{array}{c}\text { Dose de Cloro (início do } \\
\text { ensaio) } \\
\text { (mg. } \mathrm{L}^{-1} \text { ) }\end{array}$ & $\begin{array}{l}\text { Cloro residual livre } \\
\text { (final do ensaio) } \\
\left.\text { (mg. } \mathrm{L}^{-1}\right)\end{array}$ & $\begin{array}{l}\text { Remoção } \\
\text { de E2 \% }\end{array}$ & $\begin{array}{c}\text { Concentração residual } \\
\text { de E2 } \\
\left(\mu \mathrm{g} \cdot \mathrm{L}^{-1}\right)\end{array}$ \\
\hline 0,1 & 10 & 0,5 & 0,26 & 39 & 0,06 \\
\hline 1,0 & 10 & 0,5 & 0,23 & 47 & 0,41 \\
\hline 100 & 10 & 0,5 & 0,43 & 73 & 31,6 \\
\hline 1,0 & 30 & 0,5 & 0,34 & 71 & 0,26 \\
\hline 100 & 30 & 0,5 & 0,42 & 97 & 3,20 \\
\hline 100 & 10 & 1,0 & 0,83 & 82 & 20,80 \\
\hline 1,0 & 10 & 1,0 & 0,83 & 60 & 0,32 \\
\hline 1,0 & 20 & 1,0 & 0,73 & 89 & 0,11 \\
\hline 100 & 30 & 1,0 & 0,47 & 97 & 3,40 \\
\hline 100 & 30 & 1,0 & 0,52 & 94 & 5,70 \\
\hline 1,0 & 30 & 1,0 & 0,67 & 92 & 0,08 \\
\hline 0,1 & 30 & 1,0 & 0,74 & - & - \\
\hline 100 & 1 & 2,0 & 1,80 & 40 & 75,90 \\
\hline 100 & 4 & 2,0 & 1,78 & 86 & 18,20 \\
\hline 100 & 7 & 2,0 & 1,78 & 94 & 7,60 \\
\hline 100 & 10 & 2,0 & 1,79 & 97 & 3,30 \\
\hline 1,0 & 10 & 2,0 & 1,65 & 98 & $0,02^{*}$ \\
\hline 1,0 & 20 & 2,0 & 1,75 & 99 & $0,01^{*}$ \\
\hline 1,0 & 30 & 2,0 & 1,60 & 99 & $0,01^{*}$ \\
\hline 100 & 30 & 2,0 & 1,57 & 98 & 2,70 \\
\hline 0,1 & 30 & 2,0 & 1,71 & - & - \\
\hline 100 & 30 & 5,0 & 3,76 & 98 & 2,50 \\
\hline
\end{tabular}

*Amostras abaixo do LD: as amostras foram fortificadas e seus valores foram estimados; $\mathrm{n}_{2}$ : número de repetições. 
Para a concentração inicial de E2 de $1 \mu \mathrm{g} . \mathrm{L}^{-1}$, chegou-se à mesma conclusão, ou seja, para a dose de 2 mg. L $^{-1}$ o tempo de contato não influenciou a degradação do $17 \beta$-estradiol e para as doses de 0,5 e 1 mg..-1 o tempo de contato influenciou a degradação de E2 ao nível de 5\% de probabilidade (Figura 2B). De acordo com o teste de Tukey, verificou-se que para o tempo de contato de 10 min todas as doses de cloro (médias) diferem entre si ao nível de $5 \%$ de probabilidade. Para o tempo de contato de $30 \mathrm{~min}$, as médias dos tratamentos com doses de cloro com 1 e $2 \mathrm{mg} . \mathrm{L}^{-1}$ não diferem entre si, sendo diferentes da concentração de 0,5 mg. $\mathrm{L}^{-1}$. Portanto, o tratamento mais indicado para essas condições seria $2 \mathrm{mg} . \mathrm{L}^{-1}$ e $10 \mathrm{~min}$ ou $1 \mathrm{mg} . \mathrm{L}^{-1}$ com $30 \mathrm{~min}$, com concentrações finais de 21 ng. $\mathrm{L}^{-1}$ e 75 ng. $\mathrm{L}^{-1}$, respectivamente. Contudo, apesar de serem valores estatisticamente iguais, o tratamento mais adequado seria o de 2 mg. $L^{-1}$ e 10 min, pois ocorreu uma maior degradação de E2, com uma diferença na concentração final de 50 ng. $\mathrm{L}^{-1}$ (comparado ao de tratamento com $1 \mathrm{mg} . \mathrm{L}^{-1}$ e $30 \mathrm{~min}$ ), sendo esse aumento na degradação relevante do ponto de vista ambiental e de saúde pública.

\section{Efeito da concentração inicial de $17 \beta$-estradiol e de cloro}

Na Figura 3 pode-se observar que a concentração inicial de E2 afetou a remoção do hormônio, pois quanto menor a concentração inicial menor foi a eficiência de remoção. Para as mesmas condições (dose de cloro de 0,5 mg. $\mathrm{L}^{-1}$ e tempo de contato de $10 \mathrm{~min}$ ) e considerando diferentes concentrações inicias de $17 \beta$-estradiol com 100 e $1 \mu \mathrm{g} . \mathrm{L}^{-1}$ e 100 ng. $\mathrm{L}^{-1}$, as remoções foram de 73, 47 e 39\%, respectivamente. Resumindo, para $\mathrm{C}_{0}$ de $100 \mathrm{ng} . \mathrm{L}^{-1}$ e de $1 \mu \mathrm{g} . \mathrm{L}^{-1}$, a diferença entre as duas remoções foi de $8 \%$. Considerando a concentração inicial de $100 \mu \mathrm{g} . \mathrm{L}^{-1} \mathrm{em}$ relação à de 100 ng. $\mathrm{L}^{-1}$, a diferença entre as duas remoções foi de 34\%. Portanto, as considerações feitas para a concentração de $1 \mu \mathrm{g} . \mathrm{L}^{-1}$ são mais próximas ao que ocorre no ambiente onde as concentrações estão na ordem de 50 ng.L-1 (Pereira et al., 2011). A maior remoção, que ocorreu com a dose de 0,5 mg.L${ }^{1}$, foi com $C_{0}=100 \mu \mathrm{g} \cdot \mathrm{L}^{-1}$ no tempo de contato de $30 \mathrm{~min}$ (97\%). Com $C_{0}=1 \mu \mathrm{g} \cdot \mathrm{L}^{-1}$, nessas mesmas condições, a remoção foi de $71 \%$.
Portanto, pode-se inferir que com $\mathrm{C}_{0}=100 \mathrm{ng} \cdot \mathrm{L}^{-1}$ e tempo de contato de 30 min espera-se remoção igual ou menor que a encontrada com $\mathrm{C}_{0}=1 \mu \mathrm{g} . \mathrm{L}^{-1}$ e tempo de contato de $30 \mathrm{~min}$ (71\%). Adicionalmente, a remoção com $C_{0}=100 \mathrm{ng} . \mathrm{L}^{-1}$ no tempo de contato de $10 \mathrm{~min}$ foi de $40 \%$. Desse modo, conclui-se que a dose de cloro de $0,5 \mathrm{mg} \cdot \mathrm{L}^{-1}$ não é suficiente para a remoção de $17 \beta$-estradiol em ETA.

$\mathrm{Na}$ concentração de cloro de $1 \mathrm{mg} . \mathrm{L}^{-1}$, também houve efeito da concentração inicial de E2, contudo essa diferença ocorreu apenas para a concentração de $1 \mu \mathrm{g} \cdot \mathrm{L}^{-1}$ em relação a concentração de $100 \mu \mathrm{g} . \mathrm{L}^{-1}$ (Tabela 2). A remoção para $1 \mathrm{mgCl}_{2} \cdot \mathrm{L}^{-1}$ e tempo de contato de 30 min foi de 92\% para concentração inicial de $1 \mu \mathrm{g} \cdot \mathrm{L}^{-1}$ de E2. Contudo, para a concentração de 100 ng. L $^{-1}$ de $17 \beta$-estradiol, a concentração foi menor do que o LD do equipamento que é de 40 ng. L $^{-1}$ (não foi possível utilizar o procedimento de adição padrão).

Para a concentração de cloro de $2 \mathrm{mg} . \mathrm{L}^{-1}$, também foi observado efeito da concentração inicial de E2. Adicionalmente, observa-se que esse efeito tende a diminuir com o aumento da concentração de cloro e do tempo de contato. Deborde et al. (2004) chegaram à conclusão que após $15 \mathrm{~min}$ de tempo de contato com concentração de cloro de 2 mg. $L^{-1}$ e pH de 7 a 8, 95\% de remoção seria alcançada para o estrógeno $17 \beta$-estradiol, o que também foi alcançado neste estudo para a concentração de $100 \mu \mathrm{g} . \mathrm{L}^{-1}$ e tempo de $7 \mathrm{~min}$.

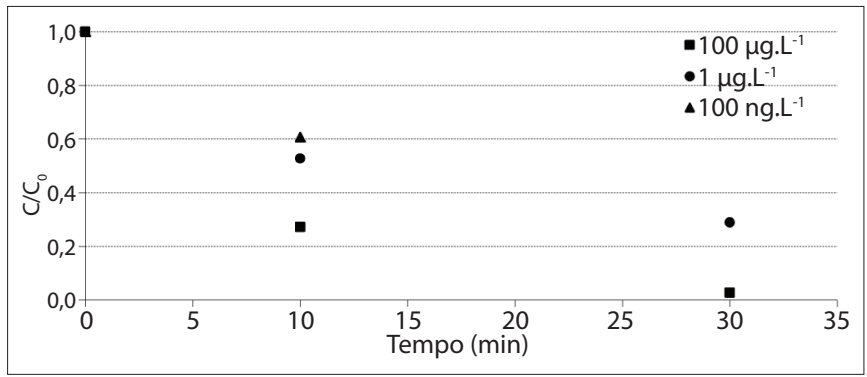

Figura 3 - Remoção de $17 \beta$-estradiol na dose de cloro de 0,5 mg.L-1 - efeito da concentração inicial de E2.
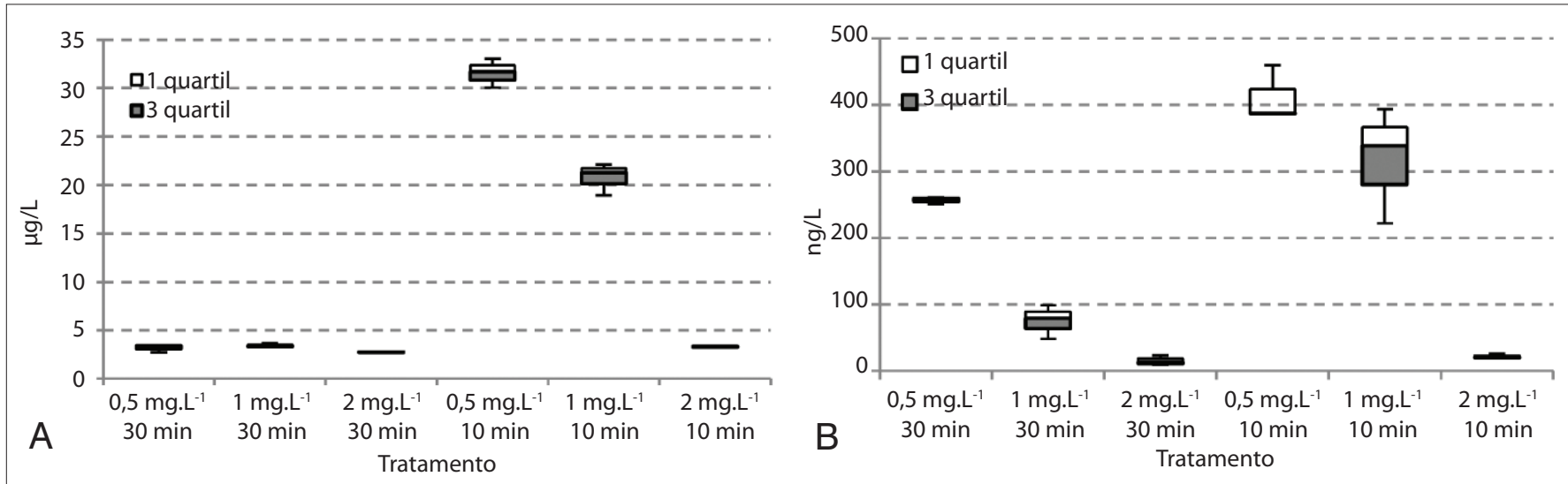

Figura 2 - Concentração residual de E2 com diferentes tempos de contato e dose de cloro considerando: (A) concentração inicial de E2 de $100 \mu$ g. ${ }^{-1}$ e (B) concentração inicial de E2 de $1 \mu \mathrm{g} \cdot \mathrm{L}^{-1}$. 
A maior remoção alcançada para a concentração inicial de $100 \mu \mathrm{g} . \mathrm{L}^{-1}$ foi de $98 \%$ com tempo de 30 min e dose de cloro de 2 mg.L $L^{-1}$, mantendo residual de $2,5 \mu \mathrm{g} . \mathrm{L}^{-1}$, suficiente para causar efeitos estrogênicos em organismos aquáticos (SUMPTER, 2005). Para a concentração de $17 \beta$-estradiol de $1 \mu \mathrm{g} . \mathrm{L}^{-1}$, a maior remoção, de $99 \%$, foi obtida com tempo de contato de 30 min e dose de cloro de $2 \mathrm{mg} \cdot \mathrm{L}^{-1}$, com um residual de E2 de 10 ng. $\mathrm{L}^{-1}$. Se for inferido que a mesma remoção ocorrerá com

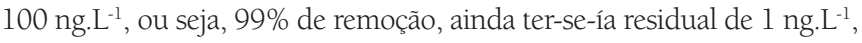
pois no tratamento com concentração inicial de E2 de 100 ng..-1 não foi possível detectar a concentração remanescente de E2. Outro fato a ser observado na Tabela 2 é que para a dose de cloro de $2 \mathrm{mg} \cdot \mathrm{L}^{-1} \mathrm{o}$ aumento do tempo de contato levou ao aumento na remoção de $17 \beta$-estradiol.

Na Figura 4, verifica-se que com o aumento da dose de cloro a eficiência de remoção de E2 aumenta. Também é possível confirmar o efeito da concentração inicial de E2, pois, para a mesma concentração de cloro e mesmo tempo de contato, há mais de 3 diferentes eficiências de remoção, sendo esse efeito mais acentuado para a concentração de 0,5 e 1,0 mg. $\mathrm{Cl}_{2} \mathrm{~L}^{-1}$.

Para verificar se a eficiência aumentaria em doses elevadas de cloro, realizou-se um ensaio com a dose de $5 \mathrm{mg} . \mathrm{L}^{-1}$ (Tabela 2). A remoção entre as doses de cloro de $5 \mathrm{mg} \cdot \mathrm{Cl}_{2} \cdot \mathrm{L}^{-1}$ e $2 \mathrm{mg}$. $\mathrm{Cl}_{2} \cdot \mathrm{L}^{-1}$ foram muito parecidas, sendo o residual de $17 \beta$-estradiol de 2,50 e 2,70 $\mu \mathrm{g}$. $\mathrm{L}^{-1}$, respectivamente. Portanto, o aumento da dose de cloro acima de $2 \mathrm{mg} \cdot \mathrm{Cl}_{2} \cdot \mathrm{L}^{-1}$ não resultou em aumento na eficiência de remoção do $17 \beta$-estradiol.

Destaca-se que para as amostras que estiveram abaixo do LD e foram fortificadas é recomendável o uso de detectores mais sensíveis para a confirmação da concentração de E2 na amostra.

\section{Avaliação do tempo na remoção de $17 \beta$-estradiol}

Para se avaliar como o aumento do tempo de contato influenciaria a remoção do $17 \beta$-estradiol, realizou-se um ensaio com tempo de contato de 24 h, como está demonstrado na Figura 5.

Constata-se que a remoção de E2 com o cloro, nos primeiros 4 min, foi de 86\%, após $10 \mathrm{~min}, 97 \%$, após $30 \mathrm{~min}$, 98\% e depois desse tempo a remoção foi lenta, chegando a 99,89\% após 12 h de reação, com concentração residual de 135,7 ng. $\mathrm{L}^{-1}$. Após 24 h de tempo de contato, o E2 não foi detectado (Figura 5). Alum et al. (2004), com concentração inicial de

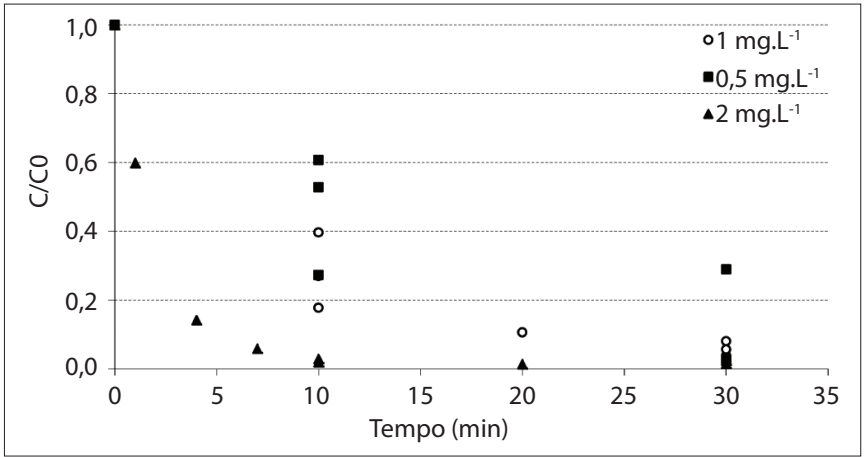

Figura 4 - Efeito da concentração de cloro na remoção de 17 $\beta$-estradiol resultados de todas as concentrações iniciais de E2.
27,23 $\mu \mathrm{g}$. $\mathrm{L}^{-1}$ após $1 \mathrm{~h}$ de tratamento com $1 \mathrm{mg} . \mathrm{Cl}_{2} \cdot \mathrm{L}^{-1}$, encontraram que o E2 estava abaixo do limite de detecção ( $\mathrm{LD}=313 \mathrm{ng} \cdot \mathrm{L}^{-1}$ ) com remoção $\geq 98,8 \%$, muito próxima à obtida neste estudo, no qual, após uma hora de tempo de contato e dose de cloro de 2,0 mg.L $\mathrm{L}^{-1}$, a remoção foi de 98,1\%. Westerhoff et al. (2005) avaliaram a remoção de E2 e chegaram à remoção de aproximadamente 100\% (< LD) após 24 h de tempo de contato, com concentração inicial de E2 de 10 a 250 ng.L.-1 e com dose de cloro de 3,8 a 3,5 mg. $\mathrm{L}^{-1}$, o que condiz com o ensaio realizado neste estudo após tempo de contato de $24 \mathrm{~h}$.

Apesar da remoção eficiente do E2, houve a formação de novos picos no cromatograma, o que indica a formação de produtos do E2, sendo que esse fato deve ser analisado por meio de detectores mais sensíveis para verificar quais são esses compostos e em qual dose de cloro a oxidação é suficiente para remover o E2 a nível não detectável. Alum et al. (2004), estudando a estrogenicidade do 17ß-estradiol, encontrou que após 4 dias de tempo de contato com o cloro (doses de cloro de 0,05 a 0,5 mg. $\mathrm{L}^{-1}$ ) ainda havia potencial estrogênico nas amostras, possivelmente dos produtos formados durante a cloração, utilizando linhagem de células humanas de câncer de mama (MCF-7). No experimento com 24 h de tempo de contato com dose de cloro de $1 \mathrm{mg} . \mathrm{L}^{-1}$, a remoção da estrogenicidade ocorreu na primeira $1 \mathrm{~h}$, permanecendo até o fim do tratamento, o que corrobora com este estudo em relação à possibilidade de formação de produtos do $17 \beta$-estradiol pelo cloro.

\section{Cinética de reação}

Tentou-se aplicar aos resultados obtidos as cinéticas de reação de pseudo-primeira ordem e pseudo-segunda ordem. Porém os desvios foram significativos, como pode ser observado na Tabela 3 através por meio do $\mathrm{r}^{2}$. As constantes cinéticas (k) para as doses de 0,5, 1 e $2 \mathrm{mg} \cdot \mathrm{L}^{-1}$ de cloro foram muito próximas entre si, considerando a cinética de pseudo-primeira ordem, contudo não se pode concluir devido à discrepância dos valores encontrados de $\mathrm{r}^{2}$ entre esses dados. Tentou-se o ajuste a outros modelos cinéticos, considerando inclusive as concentrações de cloro residual, no entanto o ajuste entre os dados observados e esperados também não foi satisfatório. Desse modo, dividiu-se em dois momentos o tratamento de $2 \mathrm{mg} . \mathrm{L}^{-1}$ e $100 \mu \mathrm{g} . \mathrm{L}^{-1}$, conforme pode ser observado na Figura 6. Assim sendo, obteve-se uma cinética de pseudo-primeira

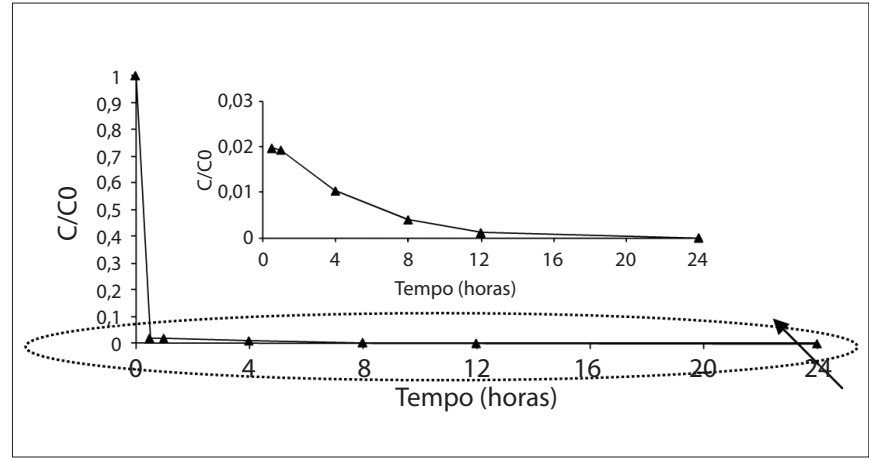

Figura 5 - Avaliação do tempo de contato na degradação de $17 \beta$-estradiol $\operatorname{com~} \mathrm{C}_{0}=2,0 \mathrm{mgCl}_{2} \cdot \mathrm{L}^{-1} \mathrm{e} \mathrm{C}_{0}=100 \mu \mathrm{g} \cdot \mathrm{L}^{-1}$ de $\mathrm{E} 2$. 
ordem: primeiro avaliou-se a cinética de reação entre o tempo de 0 a 10 min de tratamento e depois entre o tempo de 10 a 720 min de tratamento, obtendo $r^{2}$ de 0,97 e 0,99 respectivamente. De posse desses resultados, verifica-se na Figura 6 que a velocidade da reação muda drasticamente após os primeiros $10 \mathrm{~min}$, passando a uma velocidade de reação muito mais lenta do que a inicial (Tabela 3). Deborde et al. (2004), em estudo sobre a cinética de oxidação do E2 pelo cloro, chegaram a uma reação de segunda ordem, contudo considerando diferentes valores de $\mathrm{pH}(3,5$ a $12 \mathrm{pH})$ e tempos de contatos menores (de até $30 \mathrm{~min}$ ).

\section{Concentrações ambientais}

Resalvas devem ser feitas para concentrações iniciais de E2 na ordem de ng. $L^{-1}$, pois a remoção pode ser menor do que a remoção para concentrações iniciais maiores de E2. Como exemplo, no presente estudo, com tempo de contato máximo de 24 h, concentração inicial de E2 de $121 \mu g . L^{-1}$ e dose de cloro de $2 \mathrm{mg} \cdot \mathrm{L}^{-1}$, após 8 horas de reação, a remoção de E2 foi de 99,6\%, com concentração final de 484 ng.L-1. Se for considerada essa concentração como inicial e a dose de cloro nesse momento,

Tabela 3 - Constantes experimentais obtidas na degradação do E2.

\begin{tabular}{|c|c|c|c|c|c|}
\hline \multicolumn{2}{|l|}{ Tratamento } & \multicolumn{2}{|c|}{$\begin{array}{l}\text { Pseudo-1 }{ }^{\text {aoor- }} \\
\text { dem }\end{array}$} & \multicolumn{2}{|c|}{ Pseudo-2a ordem } \\
\hline $\mathrm{C}_{0}$ de E2 & $\mathrm{C}_{0}-\mathrm{Cl}_{2} \mathrm{mg} \cdot \mathrm{L}^{-1}$ & $\begin{array}{c}k^{*} \\
\left(\min ^{-1}\right)\end{array}$ & $\mathrm{r}^{2}$ & $\begin{array}{c}\mathrm{k}^{*} \\
\left(\mathrm{~L} \cdot \mu \mathrm{g}^{-1} \cdot \mathrm{min}^{-1}\right)\end{array}$ & $r^{2}$ \\
\hline 100 e $1 \mu \mathrm{g} \cdot \mathrm{L}^{-1}$ & 2,0 & 0,085 & 0,543 & 1,53 & 0,247 \\
\hline 100 e $1 \mu \mathrm{g} \cdot \mathrm{L}^{-1}$ & 1,0 & 0,081 & 0,800 & 0,136 & 0,063 \\
\hline 100 e $1 \mu \mathrm{g} \cdot \mathrm{L}^{-1}$ & 0,5 & 0,080 & 0,497 & 0,043 & 0,148 \\
\hline $\begin{array}{l}100 \mu \mathrm{g} \cdot \mathrm{L}^{-1} \\
\text { (até } 10 \mathrm{~min} \text { ) }\end{array}$ & 2,0 & 0,332 & 0,972 & 0,032 & 0,911 \\
\hline $\begin{array}{l}100 \mu \mathrm{g} \cdot \mathrm{L}^{-1} \\
(10 \text { a } 720 \mathrm{~min})\end{array}$ & 2,0 & 0,004 & 0,990 & 0,008 & 0,845 \\
\hline
\end{tabular}

$\mathrm{k}^{*}$ : velocidade específica da reação.

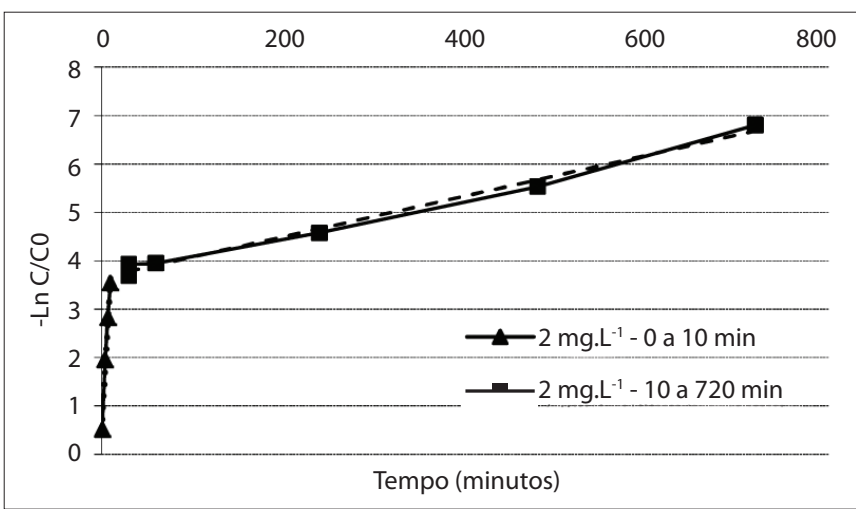

Figura 6 - Estudo da cinética da reação $-\mathrm{C}_{0}=2,0 \mathrm{mg} \cdot \mathrm{Cl}_{2} \cdot \mathrm{L}^{-1} \mathrm{e}$ $\mathrm{C}_{0}=121 \mu \mathrm{g} \cdot \mathrm{L}^{-1}$ de $\mathrm{E} 2$. que era de $1,5 \mathrm{mg} \cdot \mathrm{Cl}_{2} \mathrm{~L}^{-1}$, a remoção que ocorreu nas próximas $4 \mathrm{~h}$ de reação foi de apenas 72\%, chegando à concentração final de 136 ng. $\mathrm{L}^{-1}$. Chen et al. (2007), ao avaliarem o efeito da pré-cloração na remoção de E2 com concentração inicial de 100 ng.. L $^{-1}$, obtiveram remoções da ordem de $20 \%$ com dose de cloro de $1 \mathrm{mg}$. $\mathrm{L}^{-1}$ e tempo de contato de $10 \mathrm{~min}$, e para uma concentração inicial de E2 de 500 ng. L $^{-1}$ a remoção foi de $40 \%$. Para a pós-cloração, a remoção de E2 foi de 23\% e 36\% para as concentrações iniciais de 100 e 500 ng. $\mathrm{L}^{-1}$, respectivamente, nas mesmas condições.

Gerolin (2008), estudando a presença de E2 em amostras de água bruta e tratada de duas ETA situadas no Brasil, encontrou E2 em concentrações de 5,25, 6,66 e 7,27 ng.L-1 na água bruta de uma das ETA e no fim do tratamento a remoção total foi de 71,8, 84,7 e $80 \%$, sendo que nessa ETA a concentração de cloro residual era de 2,5 a 4,5 mg. $\mathrm{L}^{-1}$. Na outra ETA, a concentração inicial de E2 era de 3,0, 4,31 e 9,07 ng. L $^{-1}$, com remoção de 74\%, 78\% e 88,4\%, com cloro residual de 3,3 a 3,9 mg. $\mathrm{L}^{-1}$, sendo que nessa ETA é feita a pré e a pós-cloração e ainda a adição de carvão ativado. A concentração final de E2 nas duas ETA variou de 1,48 a 0,78 ng. $\mathrm{L}^{-1}$. Considerando apenas as remoções encontradas neste estudo, $92 \%$ - dose de cloro de $1 \mathrm{mg}$. $\mathrm{L}^{-1}$ e tempo de contato de $30 \mathrm{~min}$ - e $99 \%$ - dose de cloro de $2 \mathrm{mg}^{\mathrm{L}^{-1}}$ e tempo de contato de 30 min - e que a água bruta afluente a ETA contenha E2 em concentração de 10 ng. $\mathrm{L}^{-1}$, valores comumente encontrados em águas superficiais (BENOTTI et al., 2009; CHEN et al., 2007; SODRÉ et al., 2007), a concentração final na água tratada seria de 0,8 e 0,1 ng. $\mathrm{L}^{-1}$, respectivamente, o que, em teoria, condiz com o encontrado por Gerolin (2008).

\section{Conclusões}

Tendo em vista as baixas concentrações de E2 encontradas em água bruta afluente às estações de tratamento de água, aliadas à menor velocidade de reação com o cloro em baixa concentração de E2, ou devido à diminuição da concentração de E2 causada pelo aumento do tempo de reação (tempo de contato), conclui-se que a cloração não é suficiente para a completa remoção de E2 em ETA, Contudo, constatou-se que a cloração contribuiu para a remoção de E2 nas doses de cloro e tempos de contato comumente aplicados nas ETA. Devido a esse fato, e confirmando o exposto, o hormônio $17 \beta$-estradiol vem sendo detectado, mesmo que raramente, em efluentes de ETA. Portanto, quando E2 estiver presente, outros tratamentos devem ser utilizados para a sua completa eliminação. Adicionalmente, recomenda-se a realização de ensaios específicos para os estudos cinéticos dessas reações para confirmar os resultados encontrados neste estudo.

\section{Referências}

ALUM, A.; YOON, Y.; WESTERHOFF, P.; ABBASZADEGAN, M. (2004) Oxidation of bisphenol A, 17 $\beta$-estradiol, and $17 \alpha$-ethynylestradiol and byproduct estrogenicity. Environmental Toxicology, v. 19, p. 818-824.
AGÊNCIA NACIONAL DE VIGILÂNCIA SANITÁRIA. (2003) Guia para Validação de Métodos Analíticos e Bioanalíticos. Resolução-RE n 899, de 29 de maio de 2003. 
AMERICAN PUBLIC HEALTH ASSOCIATION/AMERICAN WATER WORKS ASSOCIATION/WATER ENVIRONMENT FEDERATION. (2005) Standard Methods for the Examination of Water and Wastewater. 20th, Washington DC, USA.

BENOTTI, M.J.; TRENHOLM, R.A.; VANDERFORD, B.J.; HOLADY, J.C.; STANFORD, B.D.; SNYDER, S.A. (2009) Pharmaceuticals and endocrine disrupting compouds in U.S. drinking water. Environmental Science \& Technology, v. 43, p. 597- 603.

BERTHOUEX, P.M.; BROWN, L.C. (2002) Statistics for Environmental Engineers. 2 ed. Boca Raton, FL: Lewis Publishers. p. 489.

BIRKETT, J.W.; LESTER, J.N. (2002) Endocrine disrupters in wasterwater and sludge treatment processes. Boca Raton, FL: Lewis Publishers. p. 295.

COMPANHIA DE TECNOLOGIA DE SANEAMENTO AMBIENTAL. (1987) Guia de coleta e preservação de amostras de água, São Paulo, p. 150.

CHEN, C-Y.; WEN, T-Y.; WANG, G-S.; CHENG, H-W.; LIN, Y-H.; LIEN, G-W. (2007) Determining estrogenic steroids in Taipei waters and removal in drinking water treatment using highflow solid-phase extraction and liquid chromatography. Science of the Total Environment, v. 378, p. 352-365.

CHEN, X.; HU, J. (2009) Degradation of $17 \beta$-estradiol and its conjugates: Effects of initial concentration and MLSS concentration. Process Biochemistry, v. 44, n. 12, p. 1330-1334

DEBORDE, M.; RABOUAN, S.; GALLARD, H.; LEGUBE, B. (2004) Aqueous Chlorination Kinetics of Some endocrine disruptors. Environment Science Technology, v. 38, p. 5577- 5583.

GEROLIN, E.R.R. Ocorrência e Remoção de Disruptores Endócrinos em Águas Utilizadas para Abastecimento Público de Campinas e Sumaré São Paulo. 2008. 185 f. Tese (Doutorado em Engenharia Civil) Universidade Estadual de Campinas, Campinas, Brasil, 2008

GILMAN, A.G.; HARDMAN, J.E.; LIMBIRD, L.E. (2003) As bases farmacológicas da terapêutica. 10 ed. Rio de Janeiro: Mc Graw Hill.

IKEHATA, K.; NAGHASHKAR, N.J.; EL-DIN, M.G. (2006). Degradation of Aqueous Pharmaceuticals by Ozonation and Advanced Oxidation Processes; A Review. Ozone Science e Engineering, v. 28, p. 353-414.

JURGENS, M.D.; HOLTHAUS, K.I.E.; JOHNSON, A.C.; SMITH, J.J.L.; HETHERIDGE, M.; WILLIAMS, R. J. (2002) The Potential for Estradiol and Ethinylestradiol degradation in English Rivers. Environmental Toxicology and Chemistry, v. 21, n. 3, p. 480-488.

KUSTER, M.; AZEVEDO, D.A.; LÓPEZ DE ALDA, M.J.; AQUINO NETO, F.R.; BARCELÓ, D. (2009) Analysis of phytoestrogens, progestogens and estrogens in environmental waters from Rio de Janeiro (Brazil). Environment International, v. 35, p. 997-1003.

LAGANÀ, A.; BACALONI, A.; DE LEVA, I.; FABERI,A.; FAGO, G.; MARINO, A. (2004) Analytical methodologies for determining the occurrence of endocrine disrupting chemicals in sewage treatment plants and natural waters. Analytica Chimica Acta, v. 501, p. 79-88.

LEE, H.B.; LIU, D. (2002) Degradation of $17 \beta$-estradiol and its metabolites by sewage Bacteria. Water, Air and Soil Pollution, v. 134, p. 353-368.
LEINSTER, P.; MCINTYRE, A.E.; LESTER, J.N.; PERRY, R. (1981) Analysis of volatile organic compounds in water, wastewater and an industrial effluent. Chemosphere, n. 10, p. 291.

MAIA, D.; DEZOTTI, M. (2007) Desreguladores endócrinos no meio ambiente: efeitos e conseqüências. Química nova, v. 30, n. 3, p. 651-666.

MIERZWA, J.C.; AQUINO, S.F.; VERAS, L.R.V. (2009) Remoção de desreguladores endócrinos. In: PÁDUA, V.L.P. (Coord.) Remoção de microrganismos emergentes e microcontaminantes orgânicos no tratamento de água para consumo humano. Belo Horizonte: Ed. ABES, p. 251-291.

MILLS, L.J.; CHICHESTER, C. (2005) Review of evidence: Are endocrine-disrupting chemicals in the aquatic environment impacting fish populations? Science of the Total Environment, v. 343, p. 1-34.

NAKAMURA, H.; KURUTO-NIWA, R.; UCHIDA, M.; TERAO, Y. (2007) Formation of chlorinated estrones via hypochlorous disinfection of wastewater effluent containing estrone. Chemosphere, v. 66 , p. $1441-1448$

PEREIRA, R.O.; POSTIGO, C.; DE ALDA, M.L.; DANIEL, L.A.; BARCELÓ, D. (2011) Removal of estrogens through water disinfection processes and formation of by-products. Chemosphere, v. 82, p. 789-799.

PEREIRA, R.O.; DANIEL, L.A.; DE ALDA, M.L.; BARCELÓ, D. (2012) Remoção de estrona e estradiol pelo cloro com elevado tempo de contato. In: Simpósio Luso-Brasileiro de Engenharia Sanitária e Ambiental, 15, Belo Horizonte. Anais. Belo Horizonte (MG), Brasil: Associação Portuguesa dos Recursos Hídricos, Associação Portuguesa de Engenharia Sanitária e Ambiental, Associação Brasileira de Engenharia Sanitária e Ambiental.

RODRIGUEZ-MOZAZ, S.; LOPEZ DE ALDA, M.J.; BARCELÓ, D. (2004) Picogram per liter level determination of Estrogens in Natural Water and Waterworks by a Fully automated On-Line Solid-Phase Extraction-Liquid Chromatography-Electrospray Tandem Mass Spectrometry method Analytical Chemistry, v. 76, p. 6998-7006.

SODRÉ, F.F.; MONTAGNER, C.C.F.; LOCATELLI, M.A.; JARDIM, W.F. (2007) Ocorrência de interferentes endócrinos e produtos farmacêuticos em águas superficiais da região de Campinas (SP, Brasil). Journal of the Brazilian Society of Ecotoxicology, v. 2, n. 2, p. 187-196.

SUMPTER, J.P. (2005) Endocrine disrupters in the aquatic environment: an overview. Acta Hydrochimica et Hydrobiologica, v. 33, p. 9-16.

SVENSON, A.; ALLARD, A.; MATS E.K. (2003) Removal of estrogenicity in Swedish municipal sewage treatment plants. Water Research, v. 37, p. $4433-4443$

TERNES, T.A.; STUMPF, U,M.; MUELLER, J.; HABERER, K.; WILKEN, R.-D.; SERVOS, M. (1999) Behavior and occurrence of estrogens in municipal sewage treatment plants-I. Investigations in Germany, Canada and Brazil. Science of the Total Environment, v. 225, p. 81-90.

WESTERHOFF, P.; YOON, Y.; SNYDER, S.; WERT, E. (2005) Fate of endocrine-disruptor, pharmaceutical, and personal care product chemicals during simulated drinking water treatment processes. Environmental Science \& Technology, v. 39, p. 6649-6663. 